\title{
"The Kitchen of the Revolution» \\ Food and Working-class memory in the Bicocca Area (Milan, Italy)
}

\author{
Luca Rimoldi \\ PhD in Anthropology \\ Università degli Studi di Milano Bicocca \\ Email rimoldi.luca@gmail.com
}

\section{Doi:10.5901/mjss.2013.v4n3p778}

\begin{abstract}
Human and social studies on food have underlined the link between food and identity constructions and the role of food consumption in crafting new subjectivities. In my case-study, in addition to the "social pretext" for the creation of an ethnographic encounter, I interpret food consumption in a particular place as an attempt to build a historical (and geographical) continuity between present and the narrated past. The aim of this article is to show how, during my fieldwork research, food has been both an excuse to realize the ethnographic encounter and the vehicle to fill it with social meanings. Thus, my analysis will stress a specific connection between food consumption and working-class memory in a post-industrial area of the city of Milan (Italy) known as Bicocca. The article is divided into two sections: in the first one, I show how my presence on the ethnographic field and the consumption of food represent a part of the method to collect life stories that have fuelled my ethnography; in the second one, I focus on the value of the food consumed by some of my interlocutors in the Tuscan Trattoria "da Aldo", and on how it is linked to memory of a precise political agenda created during the Hot Autumn season at Pirelli Bicocca.
\end{abstract}

Keywords: Anthropology, Food, Food Consumption, Working-class Memory, Pirelli Industries.

\section{Introduzione}

Il quartiere Bicocca è situato nella periferia nord della città di Milano. Dall'inizio del Novecento fino agli anni '70 il quartiere ha ospitato la sede principale della multinazionale italiana della gomma Pirelli. II tramonto dello sviluppo industriale di tipo fordista, cominciato già alla fine degli anni '70, ha portato negli anni '90 alla riconversione di quasi tutte le grandi industrie manifatturiere italiane e quelle della zona attorno al quartiere Bicocca non hanno fatto eccezione.

Quella della Pirelli Bicocca era un'area industriale di $750.000 \mathrm{mq}$ composta da stabilimenti che, fino agli anni '80, occupavano oltre 15.000 dipendenti. Le fabbriche Pirelli, che erano state il principale motore di sviluppo dell'area stessa, hanno progressivamente lasciato il posto a un intero nuovo quartiere, dando luogo a una delle più grandi trasformazioni urbane d'Europa, ancora oggi in corso di completamento.

I vecchi capannoni industriali hanno lasciato spazio al moderno campus dell'Università degli Studi di Milano Bicocca e ai suoi 30.000 studenti, ad alcuni importanti centri di ricerca, a diversi edifici residenziali di grandi dimensioni, ai nuovi centri direzionali della Pirelli, della Siemens e della Deutsche Bank, al Teatro degli Arcimboldi e al Bicocca Village, un centro commerciale con cinema multisala.

Si è trattato quindi di una riconversione della vocazione produttiva e dell'asse identitario di una parte fondamentale della zona: dall'industriale al terziario avanzato, dalla grande manifattura alla grande distribuzione e all'entertainment, dai caseggiati operai ai condomini residenziali di fascia medio-alta. II declino delle attività produttive legate alle grandi industrie non ha però portato a un decremento delle attività economiche nella zona, ma a una loro trasformazione.

Si evidenzia a questo proposito una grande differenziazione tra alcune delle aree che compongono il quartiere Bicocca: nelle zone che hanno visto l'insediamento delle nuove strutture del terziario, cioè quelle più contigue all'area del nuovo quartiere, vi è stata una crescita delle imprese che producono beni o servizi. Molte di queste sono legate alle attività di ricerca dell'università, ma vi è stato anche un vivace sviluppo di nuove attività di tipo commerciale, spesso nate a scapito dei vecchi esercizi al dettaglio rivolti alla popolazione operaia. Contestualmente all'insediamento delle nuove attività del quartiere si è assistito alla chiusura di molti esercizi legati al passato operaio: le storiche trattorie, i bar o le latterie a gestione familiare, i circoli e la Casa del Popolo sono stati rimpiazzati da mense self-service, fast-food, locali e ristoranti di alta fascia (soprattutto nei nuovi centri commerciali della zona). 
Ho svolto il mio lavoro di campo tra il 2008 e il 2011 proprio in questo quartiere e tutt'oggi continuo a intrattenere relazioni con i miei interlocutori: un gruppo di ex-lavoratori della Pirelli Bicocca che, legati in maniera diretta 0 indiretta alla Confederazione Generale Italiana del Lavoro (CGIL) avevano preso parte alle lotte operaie della stagione 1968/69. Durante gli anni della ricerca uno dei luoghi dove spesso mi sono dato appuntamento con gli ex-lavoratori della Pirelli Bicocca è stata la Trattoria Toscana "da Aldo", che resistendo alla trasformazione del quartiere è rimasta pressoché immutata dagli anni dell'Autunno Caldo (Immagini 1 e 2).

Avevo scelto questo locale come luogo privilegiato per condurre interviste etnografiche su suggerimento di Renzo Baricelli (1934) ${ }^{1}$, uno dei miei interlocutori principali, che sabato 23 maggio 2009 mi aveva invitato a prender parte a un pranzo da lui organizzato e a cui avrebbero partecipato «tutti i vecchi compagni della Bicocca». II locale storico del quartiere sito in Viale Sarca 187 e aperto nel 1950 da Edoardo Stipiti (detto Aldo) e da sua moglie Elsa², entrambi originari di Ponte Buggianese (Pistoia) è ora gestito dai figli della coppia: Luciano (1948) e Alberto (1962). La lunga frequentazione di questa osteria mi ha portato a riflettere sul ruolo del consumo del cibo nella costruzione di un discorso etnografico sulla memoria del quartiere stesso. In questo articolo mostrerò come il cibo sia stato uno degli elementi cruciali per la creazione di una continuità temporale tra il presente e le narrazioni dei discorsi sulla memoria operaia del quartiere Bicocca di Milano. Ciò che mi propongo di fare è, pertanto, mostrare come il cibo sia stato sia il pretesto per concretizzare l'incontro etnografico, sia il veicolo per riempire di contenuto tale incontro.

La commensalità, l'atto di "magiare insieme" è culturalmente connotato come gesto di condivisione e di creazione di un vincolo fra quelli che condividono il pasto; detti popolari da Nord a Sud dell'Italia contengono tracce di questo concetto: «Non ho mica mangiato la polenta con te» (detto lombardo), o «non sei mai venuto a mangiare a casa mia» (detto pugliese), sottolineano con la mancanza della condivisione di un pasto l'assenza di un vincolo, di un legame fra gli interlocutori.

Le questioni connesse al cibo, al suo consumo e al legame che emerge con la memoria sono state lungamente trattate, in maniera implicita o esplicita, dalla letteratura antropologica (Mintz, Du Bois, 2002; Holtzaman, 2006). Gli studi umani, negli ultimi anni, sono andati a concentrarsi sul legame che, a livello sociale, si instaura tra il cibo e la costruzione dell'identità, sia del singolo che del gruppo, sia dall'interno che dall'esterno e su come, nella società contemporanea, il cibo sia inteso come modalità di creazione di nuove soggettività (Douglas, 1985; Appadurai, 1996; Lupton, 1996; Goody, 1998 La Cecla, 1998; Montanari, 2004, Barberani, 2012).

Nel mio caso, invece, oltre al "pretesto sociale" per la creazione di un incontro etnografico, interpreto il consumo del cibo in un determinato luogo come tentativo di costruzione di una continuità storica (oltre che geografica) tra il presente vissuto e il passato narrato. Come sostiene Coleman: «Oltre all'atto di mangiare ci sono poche aree della vita sociale in cui l'esperienza dell'etnografo è più immediata e incorporata e dove l'apprendimento culturale richiede un maggior impegno. Il cibo è il primo medium attraverso cui ogni persona, dall'infanzia, impara a creare e a gestire le relazioni con il mondo esterno e con le altre persone che lo popolano» (Coleman, 2013: 1-2).

L'articolo è diviso in due parti. Nella prima mostrerò come il mio posizionamento sul campo e il consumo del cibo abbiano rappresentato, negli anni di ricerca, una parte del metodo di raccolta delle storie di vita che hanno alimentato la mia etnografia. Attraverso la commensalità ho potuto, infatti, avere una serie di conversazioni che sono andate articolando il discorso sulla memoria operaia che ho portato avanti (Rimoldi, 2010; 2011). Nella seconda parte, invece, mi concentro sul valore del cibo consumato dai miei interlocutori nella trattoria toscana "da Aldo", e su come esso si leghi al ricordo di una precisa agenda politica portata avanti durante la stagione dell'Autunno Caldo alla Pirelli.

\section{Posizionamento e consumo del cibo}

Nella parte iniziale del primo dei sette libri che compongono "Alla Ricerca del Tempo Perduto", Proust suggerisce che attraverso le sensazioni derivate dal mangiare una madeleine inzuppata nel tè tornino alla mente del protagonista i ricordi della sua infanzia, i luoghi in cui era cresciuto, prendano forma le figure di allora così come le sue emozioni e i suoi sentimenti. II sapore e la fragranza della madeleine evocano sensazioni in grado di attivare la memoria inconscia del protagonista, a riprova che l'atto del mangiare non possa essere considerato un gesto neutro rispetto al percorso di costruzione del sé.

Se, come ho sostenuto altrove, il "campo narrato" si è andato costruendo attraverso il mio posizionamento sul campo e la relazione che si è instaurata con le persone che hanno partecipato alla mia ricerca (Rimoldi, 2012), qui

${ }^{1}$ Renzo Baricelli è stato rappresentante sindacale per la CGIL durante la stagione dell'Autunno Caldo in Bicocca.

${ }^{2}$ A Elsa Stipiti il gruppo musicale "Gli Esuli" ha dedicato la canzone "Trattoria da Aldo" contenuta nel cd "Caldarroste e Gelati" di cui parlerò nel corso della mia argomentazione. 
descriverò proprio alcuni degli elementi che hanno autorizzato la relazione stessa e cercherò di isolare alcuni dei livelli di significato ad essi attribuiti. Tra queste, senza dubbio, il consumo del cibo e la commensalità meritano certamente un'attenzione particolare se consideriamo l'esperienza intima del lavoro di campo come fonte primaria di conoscenza. II mio lavoro etnografico, infatti, mi ha suggerito che, al di là della vicinanza geografica del contesto di analisi, il consumo del cibo durante gli incontri etnografici non abbia solamente a che vedere con la nutrizione 0 con la soddisfazione di bisogni meramente biologici ma che esso si leghi profondamente al luogo e al tempo in cui esso viene consumato. Nel mio caso la dimensione temporale viene ampliata e il consumo di un determinato cibo in un determinato luogo viene letto come una pratica per rinsaldare il legame tra i membri di uno stesso gruppo e il loro comune passato. Per rendere conto della varietà delle singole esperienze all'interno del quadro sociale di una memoria condivisa su cui i miei interlocutori hanno riflettuto nel presente introduco il concetto etico di "generazione effimera". Considerando le narrazioni dei miei interlocutori ho infatti notato che gli avvenimenti storici con cui le narrazioni delle storie di vita si intersecano sono già accaduti, sono già stati ripensati e analizzati da diversi punti di vista, sono stati mitizzati e sono entrati a far parte di tutta una serie di retoriche in materia di lavoro, di diritto al lavoro, di mobilitazione e di lotta politica (Rimoldi, 2013).

Con l'espressione "campo narrato" faccio invece riferimento a due aspetti che hanno caratterizzato il mio lavoro di ricerca: il primo è che, contrariamente a quanto solitamente avviene durante la pratica del lavoro etnografico, la realtà che ho osservato e che ho cercato di documentare non è "in atto" e dunque non può essere osservata e analizzata attraverso il più classico metodo antropologico dell'osservazione etnografica; le descrizioni del quartiere, così come quelle della fabbrica e di ciò che è successo nel periodo storico significativo preso in considerazione trovano una ragion d'essere solamente all'interno di conversazioni, racconti, materiali d'archivio da me raccolti negli anni di ricerca sul campo. In altri termini, la morfologia del mio campo si è andata creando e articolando nel mio lavoro di ricerca seguendo le traiettorie storiche riportate nei racconti dei miei vari interlocutori siano essi i materiali di archivio o le storie di vita.

II secondo aspetto che giustifica l'utilizzo dell'espressione "campo narrato", strettamente legato al primo, fa riferimento al fatto che ho cercato di far confluire nel mio lavoro diversi tipi di narrazioni, con prospettive diverse e raccontate da diversi punti di vista, in grado di mostrare la complessità dei rapporti tra presente e passato e di sottolineare come le fonti ricostruite nel presente siano in grado di gettare un'ulteriore luce sul passato.

Come dicevo, il mio accesso al campo narrato e il mio posizionamento all'interno di questo spazio è avvenuto proprio tra le pareti della trattoria toscana "da Aldo" durante il già ricordato pranzo organizzato da Renzo Baricelli. Quel giorno, infatti, Vito Basilico aveva con sé un'audiocassetta contenente un'intervista che aveva rilasciato a Radio Popolare qualche anno prima (1999): «In questa cassetta qui io racconto la storia dei tre giorni al grattacielo Pirelli e dimostro che già da lì si potevano vedere le avvisaglie di quello che sarebbe successo dopo. Quelli del mio partitino [Partito Comunista dei Lavoratori] vorrebbero mettere il testo su internet ma nessuno ha il tempo, la voglia e la pazienza, di mettersi lì con il registratore e trascriverne il contenuto». Avevo avuto in prestito la cassetta in cambio della promessa di produrre una copia del nastro e, quando la settimana successiva tornai da Aldo per incontrare Vito Basilico e per restituirgli la cassetta originale e la copia, portai con me anche la trascrizione (cartacea e digitale) della sua intervista. «Ma quel lavoro lì delle cassette è una cosa grossa....l'unica che aveva messo mano era stata una compagna del mio partitino comunista, quello del Ferrando. Con tutta la decoderia [il neologismo viene dalla parola decoder. II riferimento alla modalità di trasmissione del segnale televisivo viene dal fatto che, proprio in quel periodo, stava iniziando il passaggio da analogico a digitale] non c'è ancora lo strumento che permetta di tradurre il parlato delle cassette in scritto». Dopo avermi ringraziato Vito Basilico mi aveva dato la sua immediata disponibilità per pranzare insieme "da Aldo" e per discutere di come i suoi racconti potevano essere utili per il mio lavoro di ricerca dandomi così la possibilità di negoziare il mio ruolo di giovane ricercatore con lui e con gli altri ex-lavoratori della Pirelli Bicocca che, di tanto in tanto, frequentavano il locale. Pranzare "da Aldo" con le persone che hanno preso parte alla mia ricerca ha rappresentato sia il rito di passaggio che mi ha permesso l'accesso al campo narrato sia ciò che ha autorizzato, agli occhi dei miei interlocutori, la possibilità di scrivere le loro storie di vita e di lavoro spesso raccontatemi davanti ai piatti del menù fisso della trattoria toscana.

Tuttavia, l'entrare in relazione con i miei interlocutori non ha rappresentato l'ingresso in un mondo culinario "altro", un adattamento 0 un'integrazione dei mie gusti in fatto di cibo. Inutile rimarcare che, al di là delle specifiche preferenze alimentari individuali, condivido con i miei interlocutori tanto la lingua ${ }^{3}$ quanto, per usare l'espressione di Montanari (2010), "l'identità italiana in cucina". Ciò che, certamente, non condividevo con loro era l'esperienza del lavoro in fabbrica e delle lotte sindacali portate avanti più di quarant'anni fa che i miei interlocutori hanno cercato di ricostruire attraverso le narrazioni delle loro storie di vita e di lavoro.

\footnotetext{
${ }^{3}$ Come ricorda Marino Niola (2009) riprendendo la celebre riflessione di Claude Lévi-Strauss (1964), il rapporto che gli uomini intrattengono con l'alimentazione assomiglia a quello che instaurano con il linguaggio: entrambi ci appaiono naturali, soggetti a regole inconsce 0 apprese sin dalla nascita.
} 


\section{Il consumo del cibo come pratica di resistenza}

Le lotte operaie dell'Autunno Caldo alla Pirelli Bicocca, partendo da istanze locali, di fabbrica, hanno ben presto assunto delle connotazioni politiche di più ampio respiro. Come suggerisce il giornalista Mimmo Spina:

"La Pirelli è l'azienda simbolo delle lotte sindacali in Italia; a partire dagli anni '60 erano migliaia i lavoratori, per la maggior parte operai, che varcavano $i$ cancelli della fabbrica in una zona periferica milanese chiamata la Bicocca degli Arcimboldi. C'è stato un tempo in cui l'assemblea generale veniva fatta di fronte all'ingresso principale, nel campo sportivo la cui capienza era, però, di sole duemila persone, le altre quattro o cinque mila restavano fuori, in strada. Alcuni dei protagonisti di quegli anni si sono incontrati una domenica. Credevano di essere all'osteria e invece stavano nella storia».

Con queste parole Spina aveva introdotto il suo documentario4 "1968...C'era una volta la Pirelli-Bicocca: storie, vicende e passioni". II documentario è stato girato proprio nella Trattoria Toscana "Da Aldo". Oggi, la trattoria non è più così affollata ma certe caratteristiche sono rimaste immutate. Anche oggi le vetrine sono quasi oscurate e, sopra di esse, campeggia una semplice insegna scura. Sui tavoli, posizionati a poca distanza l'uno dall'altro e apparecchiati con tovaglie di plastica a quadri bianchi e rossi, vengono appoggiate tovagliette e tovaglioli di carta. Le pareti sono ricoperte da cornici contenenti articoli di giornale che parlano del locale e vari riconoscimenti attribuiti ad $\mathrm{Aldo}^{5} \mathrm{e}$ alla sua trattoria ${ }^{6}$. Tra le bandiere appese spiccano la bandiera di Cuba e quella della pace; gli occhi di Che Guevara guardano gli avventori del locale dalle pose più celebri mentre il profilo barbuto di un giovane Fidel Castro all'indomani della rivoluzione cubana si fa subito notare da chiunque metta piede all'interno del locale (Immagini 5 e 6 ). Recandosi in trattoria tra mezzogiorno e le tre di pomeriggio di un qualunque giorno della settimana non è difficile incontrare, seduto a uno dei tavoli e spesso circondato da altri avventori di ogni età, Vito Basilico ${ }^{7}$ (1926) che, come spesso mi ha ripetuto:

«lo questi qui [riferendosi ai fratelli Stipiti] li conosco dal 1955, da quando ho iniziato a venire a mangiare qui. Quando si aprivano i cancelli della Pirelli tutti noi venivamo qui in trattoria ma...non si trattava solo di mangiare ma di continuare a discutere di quello che dicevamo nelle riunioni» (Vito Basilico).

Lo stesso Vito Basilico ricorda che quando Aldo aveva appena aperto il suo locale si mangiavano solamente polenta e pesce fritto; il lavoro iniziava alle 5 del mattino dal momento che già alle 6 si riempiva dei turnisti che smontavano dal turno di notte e si andava avanti fino a mezzanotte, dopo aver servito i turnisti delle 22. Luciano e Alberto Stipiti, gli attuali gestori, mi hanno raccontato che inizialmente il locale era una rosticceria e occupava circa la metà della superficie che occupa ora; i loro genitori avevano col tempo ampliato il menù e gli operai e gli impiegati della Pirelli Bicocca, dopo aver ordinato il cibo, andavano a consumarlo nella vicina Casa del Popolo, a pochi passi di distanza seguendo viale Sarca in direzione sud. Poco prima dell'inizio degli anni '60 il locale inizia ad assumere l'aspetto che conserva ancora oggi: vengono collocati i primi tavolini e i clienti iniziano a consumare il cibo all'interno del locale.

Entrando nel locale durante un'assolata giornata dell'estate del 2013 Luciano mi accoglie raccontandomi un episodio accaduto qualche giorno prima:

«leri si sono affacciati due tipi che mi hanno chiesto: "ma voi siete di sinistra? -io ho riposto di sì e lui ha dettoallora andiamo a mangiare da un'altra parte, -ma a me che importa se questi vogliono andare a mangiare da un'altra parte? Noi siamo di sinistra» (Luciano Stipiti).

Questo, a mio avviso, mostra come consumare un pasto "da Aldo" ha ancora oggi un significato politico e sociale molto profondo. Rivolgendo nuovamente lo sguardo al passato Vito Basilico e Luciano Stipiti ricordano la decisione di Pirelli di spostare di qualche metro più a nord la portineria prima ubicata proprio sul lato opposto della strada rispetto al locale di

\footnotetext{
4 Il documentario di Mimmo Spina è andato in onda nel 2008 sul canale Rai News 24.

${ }^{5}$ Appesa alla colonna centrale del locale c'è la Croce di Cavaliere al merito della Repubblica per grandi meriti civili e sportivi concessa ad Aldo da Sandro Pertini, Presidente della Repubblica italiana, nel 1982.

6 II Comune di Milano ha riconosciuto il locale come una delle "botteghe storiche" del capoluogo lombardo (Immagini 3 e 4).

7 Vito Basilico, disegnatore del Laboratorio Pirelli sin dagli anni '50, è stato uno dei leader delle lotte operaie alla Pirelli Bicocca.
} 
Aldo che viene interpretato come un atto del "padrone" per evitare la socializzazione e lo scambio di idee tra gli operai e gli impiegati.

«La portineria era proprio qui di fronte, e gli operai andavano a mangiare lì a mezzogiorno, e la Pirelli ha fatto spostare la portineria qualche metro più a nord per evitare che gli operai andassero a mangiare lì, lì era una sede di dibattito e di incontro» (Vito Basilico).

Alle loro parole sembrano far eco quelle di Luigi Roma ${ }^{8} \mathrm{che}$, parlando di come erano organizzate le mense all'interno della Pirelli Bicocca sul finire degli anni '60, mi racconta:

«La divisione delle mense era un fatto politico perché se tu sei a mangiare insieme al tuo assistente, hai l'opportunità per discutere... e non si voleva che ci si parlasse troppo tra gli operai e con gli impiegati... secondo me è una mia idea. Invece, ci tenevano separati così da farci avere poche possibilità di scambiarci le opinioni. Per un impiegato... diventare amico di un operaio non era certo una buona cosa, vista dal punto di vista del padrone. L'impiegato, a volte, può dire cose, per esempio, sui progetti di produzione -dipende dal posto che occupa- perché anche i posti da impiegati sono diversi: ci sono gli impiegati tecnici e gli impiegati amministrativi che sono quelli che tengono la contabilità» (Luigi Roma).

Il consumo del cibo in sé così come il luogo in cui esso viene consumato e la socialità che si genera vengono ricordati come degli elementi di contrasto tra un "noi" -operai e impiegati che scendevano in piazza durante l'Autunno Caldo- e un "loro" -"i padroni" che cercavano in tutti i modi di mantenere il controllo sui corpi dei lavoratori affinché garantissero loro il massimo della produttività in un ambiente di lavoro spesso descritto dai miei interlocutori come malsano e, a tratti, schiavizzante ${ }^{9}$.

Nello stesso giorno Vito Basilico davanti a un bicchiere di vino rosso e a un piatto della Trattoria Toscana, sebbene non avesse perso occasione di farmi notare che, data l'età, non sarebbe stato in grado di descrivermi «l'effervescenza politica» che si andava creando, dopo aver bevuto il caffè e un bicchiere di grappa, aveva iniziato a parlare di ciò che pensa della situazione politica italiana contemporanea, di che cosa sia il socialismo e di chi siano "gli ultimi" sottolineando come per lui, un tempo alla testa degli scioperi di impiegati e operai della Pirelli Bicocca, percorrere in bicicletta la strada che separa la sua abitazione dalla Trattoria Toscana e consumare lì il pranzo quotidianamente sia una pratica di resistenza a un presente in cui fatica a collocarsi. Nelle sue stesse parole:

«lo vengo ancora qui, a mangiare da Aldo, per me è un modo di continuare a combattere, per ascoltare i discorsi dei lavoratori che vengono a mangiare qui, lontani dai posti di lavoro, e anche per ritrovare i vecchi compagni: ogni tanto ne spunta fuori qualcuno» (Vito Basilico).

La nostalgia che permea la narrazione dei ricordi del lavoro in fabbrica di Vito Basilico sembra trovare conferma nelle strofe della canzone del gruppo musicale "Gli Esuli" intitolata proprio "Trattoria da Aldo":

"Quel che tu sogni ancora/già è un ricordo del passato Elsa/ fatti una ragione/cambia in fretta una stagione/ speri ancora di vedere/affacciandoti sul viale/ tante insegne di Pirelli/ma son bianchi i tuoi capelli. Ti ricordi in Trattoria/arrivavano i turnisti/ bianche tute di cotone/ e una fame da leone. Raccontavano di ore/che il padrone mal pagava/ma azzittivano sul piatto/che Aldo preparava. Chi beveva tanto vino/chi metteva troppo sale/ c'era chi cedeva il posto/già finito di mangiare. Cara Elsa innamorata/ di quei giorni ormai Iontani/tanti amici, pochi soldi/ cara Elsa ti ricordi. Ed in trattoria da Aldo/c'era gioia, c'era caldo, c'era pronto tutto a posto/c'era trippa, c'era arrosto (...)»

Lo sguardo nostalgico di Vito Basilico, come quello degli autori della canzone ${ }^{10}$ e quello dei fratelli Luciano e Alberto Stipiti si rivolge verso il passato, verso la stagione delle lotte sindacali. Negli anni '60, mi racconta Vito Basilico, il senso

${ }^{8}$ Ex operaio del reparto 31 (cavi) e delegato sindacale dello stesso reparto.

${ }^{9} \mathrm{~A}$ tal proposito Renzo Baricelli mi aveva detto: "C'era una condizione di non libertà all'interno della fabbrica e una sofferenza nel vedersi limitati nella propria libertà di pensiero e azione sindacale e politica. Anche se c'era libertà formale era rischioso entrare in fabbrica con un giornale come "L'Unità" in tasca. C'era nel lavoratore questa percezione di vivere in un ambiente con una disciplina vessatoria, da caserma. II sistema di comando e di controllo -tramite i capi e le guardie- era tale per cui si creava nei lavoratori sofferenza anche dal punto di vista umano» (Renzo Baricelli).

10 Felice Carrauto ed Emilio Galeotta. 
di appartenenza politica appariva molto più marcato ed evidente rispetto ad oggi e, in questo senso, al consumo del cibo vengono attribuiti significati politico-sociali ben definiti che rientrano in ciò che ho considerato come parti di un discorso più ampio sulla memoria operaia del quartiere.

"Questa era la cucina della rivoluzione, qui le idee politiche che circolavano venivano alimentate con il cibo che Aldo preparava e dissetate con un bicchiere di vino rosso» (Vito Basilico).

II ruolo identitario e simbolico del consumo di un determinato cibo in un determinato luogo emerge con prepotenza nelle parole di Vito Basilico e sembra tracciare una continuità tra il suo passato e il suo presente:

«negli anni Sessanta ero alla testa di milioni di persone, ora mi ascoltano in quattro gatti ma nessuno ha ancora smontato la mia insistente voglia di cambiare il mondo» (Vito Basilico).

così come tra il presente e il passato del quartiere Bicocca stesso. Mentre Vito Basilico pronuncia queste parole, Luciano Stipiti lo guarda quasi ipnotizzato, quasi perché con la coda dell'occhio segue e dirige i movimenti del fratello Alberto: «Non dare i peperoni a Maurizio -un giovane avventore della trattoria seduto al tavolo accanto al nostro- che non li digerisce!», come se, pronunciando questa frase, mi avesse voluto ribadire che, ancora oggi, venire a mangiare "da Aldo" significa, come nei proverbi, creare un vincolo.

\section{Conclusioni}

In conclusione, ho cercato di comprendere come il consumo del cibo si sia legato a un certo tipo di costruzione identitaria e alla creazione di un ricordo nostalgico di una stagione politicamente densa di significato per la storia italiana. In particolare consumare del cibo nella trattoria toscana "da Aldo" rappresentava, negli anni delle lotte operaie alla PirelliBicocca, un tentativo di sfuggire a ciò che viene narrato come il "controllo del padrone". A conferma di questa ipotesi ci sarebbe, appunto, il tentativo di Pirelli di impedire la socialità creata dalla commensalità operaia e impiegatizia. Ho mostrato come sia il cibo consumato, per come ricordato, sia quello che viene consumato, nello stesso luogo, oggi siano profondamente collegati a un discorso più ampio sulla memoria operaia del quartiere Bicocca di Milano evidenziando il valore simbolico e identitario del cibo in sé come catalizzatore della memoria individuale e collettiva. Ho inoltre cercato di mostrare come i discorsi sul cibo assumano una dimensione profondamente politica e come il consumo del cibo sia ricordato come uno degli elementi in cui si sono esplicitate le dinamiche "operai/padrone" raccontate dagli ex-lavoratori della Pirelli. Non da ultimo, ho evidenziato anche il valore metodologico che il consumo del cibo ha assunto durante gli anni della mia ricerca sul campo.

\section{Immagini}
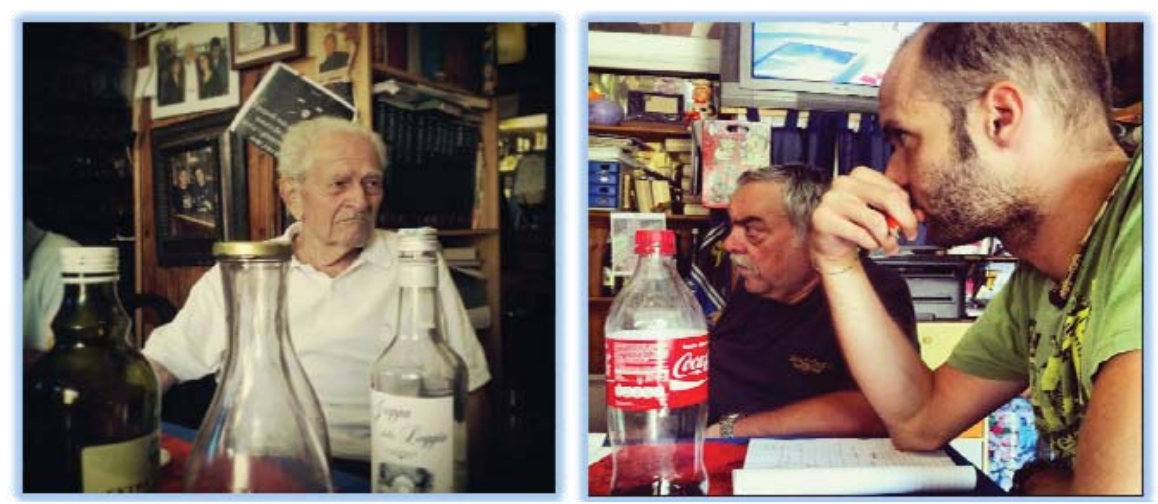

Immagini 1 e 2: Vito Basilico (1), Luciano Stipiti e Luca Rimoldi (2) durante un pranzo alla Trattoria da Aldo 

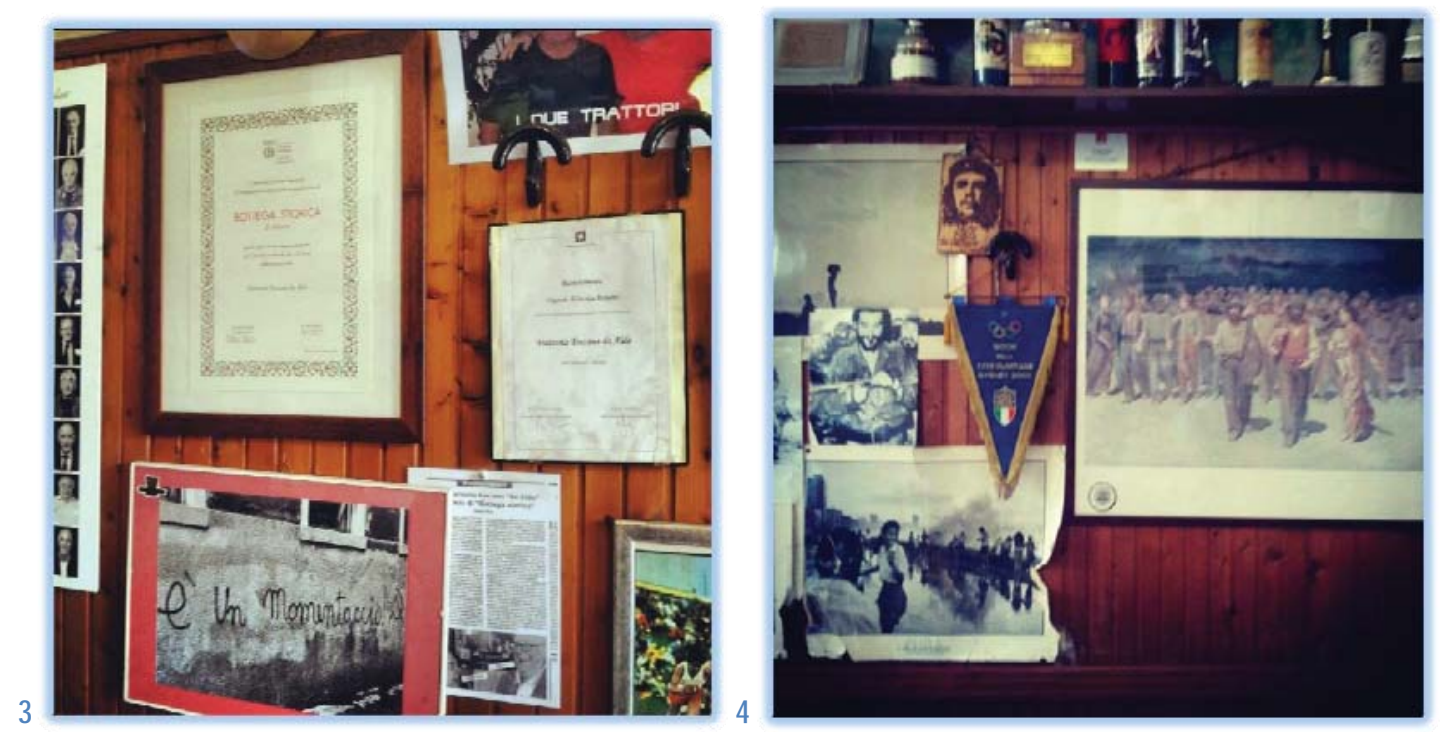

Immagini 3 e 4 : Particolari delle pareti della trattoria «da Aldo»
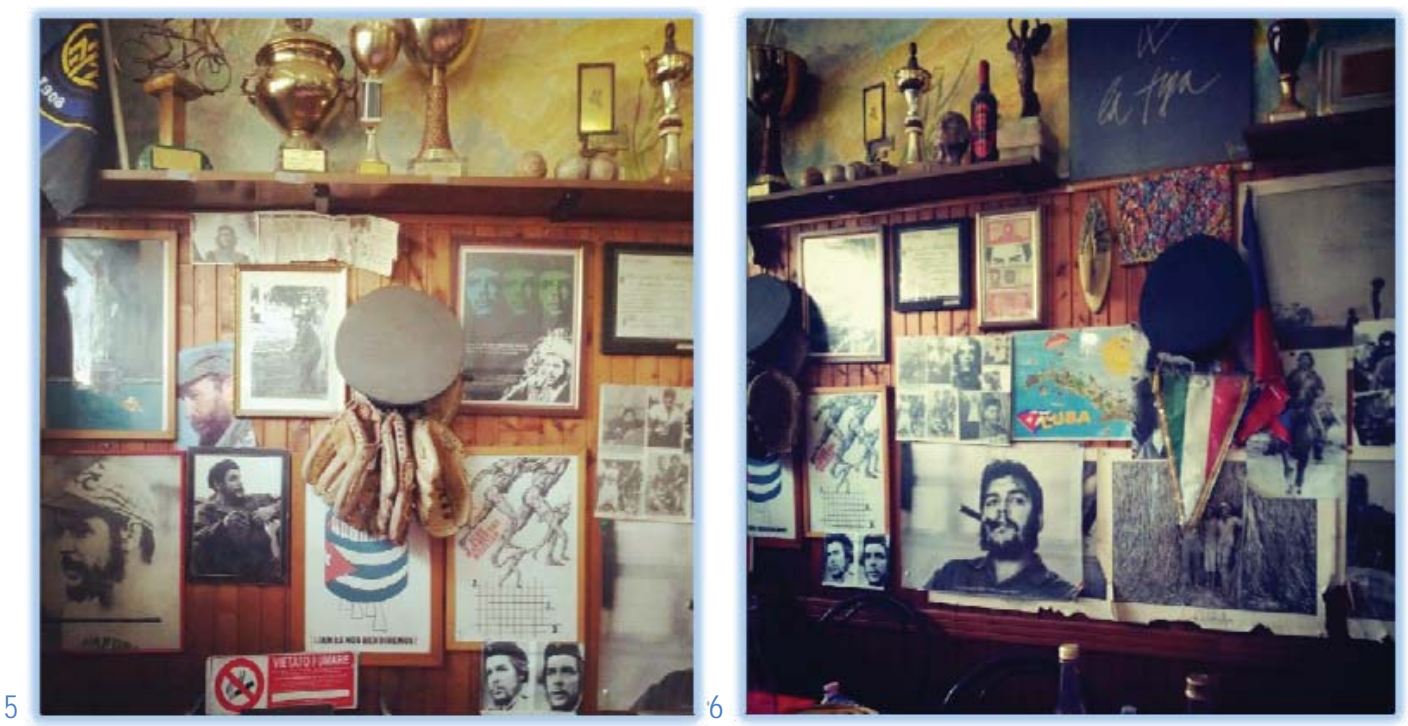

Immagini 5 e 6: Particolari delle pareti della trattoria «da Aldo»

\section{Bibliografia}

Appadurai, A., (1996). Modernity at Large: Cultural Dimensions of Globalization, Minneapolis-London: University of Minnesota Press [ed. it. (2012). Modernità in polvere, Milano: Raffaello Cortina] 
Barberani, S., (2012). The Poetics of Food Consumption: Alimentary Rhetorics and Creative Practices. Mediterranean Journal of Social Sciences Vol. 3 (7) pp. 285-291

Cercone, A., (2007). Dagli Stipiti il pranzo è servito. II Giorno, domenica 6 maggio 2007

Coleman, L., Ed. (2013). Food. Ethnographic Encounters. Oxford, New York: Berg

Douglas, M., (1985). Antropologia e Simbolismo, II Mulino: Bologna

Goody, J., (1998). Food and Love. A Cultural History of East and West, London: Verso [ed. it (2012) Cibo e amore. Storia culturale dell'Oriente e dell'Occidente, Milano: Raffaello Cortina]

La Cecla, F., (1998). La pasta e la pizza. Bologna: II Mulino

Lupton, D.,(1996). Food, the Body and the Self. London: Sage [ed.it (1998). L'anima nel piatto, Bologna: II Mulino]

Holtzman, J.D., (2006). Food and Memory. Annual Review of Anthropology Vol. 35 pp. 361-378

Lévi-Strauss, C., (1964). Mythologiques I : Le Cru et le Cuit, Paris, Plon [ed. it. (2008). II Crudo e il cotto, Milano: II Saggiatore]

Mintz, S. W.; Du Bois, C. M., (2002). The Anthropology of Food and Eating. Annual Review of Anthropology Vol. 31 pp. 99-119

Montanari, M., (2004). II cibo come cultura. Torino: Laterza

Montanari, M., (2010). L'identità italiana in cucina. Torino: Laterza

Niola, M., (2009). Si fa presto a dire cotto. Un antropologo in cucina. Bologna: II Mulino

Proust, M., (2009). Alla ricerca del tempo perduto. Roma: Newton Compton

Rimoldi, L., (2010). «Rivoluzionari di professione». Storie di sindacalisti della Cgil nella Pirelli degli anni Sessanta e Settanta. In Vignato, S., (Ed.), Soggetti al lavoro. Un'etnografia della vita attiva nel mondo globalizzato (pp. 3-17). Torino: Utet

Rimoldi, L., (2011). Sul marciapiede di viale Sarca a Milano. Storia e storie delle «battaglie» Vito Basilico, sindacalista della Pirelli. Memoria/Memorie, Special Issue Avanti Popolo. Per una storia del sindacato: Iotte, riflessioni, analisi, 6, 37, 49-72

Rimoldi, L., (2012). The Construction and the Negotiation of Ethnographic Voices Notes From an Italian Post-Industrial Area. Mediterranean Journal of Social Sciences Vol. 3 (8) pp. 221-226

Rimoldi, L., (2013). Rivoluzionari di Professione. Idee di memoria e pratiche di lavoro nella Pirelli degli anni Sessanta. Tesi di dottorato in "Antropologia della contemporaneità: etnografia delle diversità e delle convergenze culturali", Università degli studi di MilanoBicocca. 